\title{
Review
}

\section{Osseous Infections Caused by Aspergillus Species}

\author{
Christos Koutserimpas ${ }^{1}$, Ifigeneia Chamakioti ${ }^{1}$, Konstantinos Raptis ${ }^{1}$, Kalliopi Alpantaki ${ }^{2}$, Georgia Vrioni ${ }^{3} \mathbb{D}$ \\ and George Samonis ${ }^{4, *}$
}

1 Department of Orthopaedics and Traumatology, "251" Hellenic Air Force General Hospital of Athens, 11525 Athens, Greece; chrisku91@hotmail.com (C.K.); chamakiotiifigeneia@gmail.com (I.C.); kraptis1981@hotmail.gr (K.R.)

2 Department of Orthopaedics and Traumatology, "Venizeleion" General Hospital of Heraklion, 17121 Heraklion, Greece; apopaki@yahoo.gr

3 Department of Microbiology, Medical School, National and Kapodistrian University of Athens, 11527 Athens, Greece; gvrioni@med.uoa.gr

4 Department of Internal Medicine, University Hospital of Heraklion, 71500 Heraklion, Greece

* Correspondence: samonis@med.uoc.gr; Tel.: +30-6948712130

check for updates

Citation: Koutserimpas, C.; Chamakioti, I.; Raptis, K.; Alpantaki, K.; Vrioni, G.; Samonis, G. Osseous Infections Caused by Aspergillus Species. Diagnostics 2022, 12, 201. https://doi.org/10.3390/ diagnostics12010201

Academic Editors: Alfred O. Ankrah, Andor W.J.M. Glaudemans and Mike M. Sathekge

Received: 20 December 2021

Accepted: 12 January 2022

Published: 14 January 2022

Publisher's Note: MDPI stays neutral with regard to jurisdictional claims in published maps and institutional affiliations.

Copyright: (c) 2022 by the authors. Licensee MDPI, Basel, Switzerland. This article is an open access article distributed under the terms and conditions of the Creative Commons Attribution (CC BY) license (https:/ / creativecommons.org/licenses/by/ $4.0 /)$.

\begin{abstract}
Background: Osteomyelitis caused by Aspergillus spp. is a severe, but rare, clinical entity. However, clear guidelines regarding the most effective medical management have not yet been established. The present study is a literature review of all such cases, in an effort to elucidate epidemiology, as well as the therapeutic management and the infection's outcome. Methods: A thorough review of all reports of osteomyelitis of the appendicular and the axial skeleton, without the skull and the spine, caused by Aspergillus spp. was undertaken. Data about demographics, imaging techniques facilitating diagnosis, causative Aspergillus, method of mold isolation, antifungal treatment (AFT), surgical treatment, as well as the infection's outcome were recorded and evaluated. Results: A total of 63 cases of osseous infection due to Aspergillus spp. were identified. The studied population's mean age was 37.9 years. The most commonly affected site was the rib cage (36.8\%). Most hosts suffered immunosuppressive conditions (76.2\%). Regarding imaging methods indicating diagnosis, computer tomography (CT) was performed in most cases $(42.9 \%)$, followed by plain X-ray (41.3\%) and magnetic resonance imaging (MRI) (34.9\%). The most frequent isolated mold was Aspergillus fumigatus (49.2\%). Cultures and/or histopathology were used for definite diagnosis in all cases, while galactomannan antigen test was additionally used in seven cases (11.1\%), polymerase chain reaction (PCR) in four cases $(6.3 \%)$, and beta-D-glucan testing in three cases (4.8\%). Regarding AFT, the preferred antifungal was voriconazole $(61.9 \%)$. Most patients underwent surgical debridement $(63.5 \%)$. The outcome was successful in $77.5 \%$. Discussion: Osteomyelitis due to Aspergillus spp. represents a severe infection. The available data suggest that prolonged AFT in combination with surgical debridement is the preferred management of this infection, while identification of the responsible mold is of paramount importance.
\end{abstract}

Keywords: fungal ostemyelitis; osteoarticular infection; osseous infection; Aspergillus

\section{Introduction}

Fungal osteoarticular infections represent a severe invasive disease caused by hematogenous dissemination, but also by direct inoculation, or direct extension from a nearby infection focus [1-3]. Hematogenous spread is the most frequent cause of osseous infection, while direct inoculation is mainly associated with prosthesis implantation or instrumentation. The incidence of such infections is constantly increasing mainly in immunocompromised hosts $[2,3]$. Nevertheless, they also may more rarely affect immunocompetent patients as well [2]. The main predisposing factors for fungal osteomyelitis are immunosuppression by disease or medications, substance abuse, presence of an indwelling catheter, parenteral hyperalimentation, diabetes mellitus, long-term use of broad-spectrum antimicrobials, HIV infection, and organ transplantation [1,2]. 
Aspergillus spp. are ubiquitous molds causing a wide range of clinical syndromes depending on the immune status of the host [1-3]. Immunocompromised patients have increased during the last decades, leading to consecutive increases in fungal invasive infections. Invasive Aspergillosis represents an important cause of morbidity as well as mortality in immunocompromised patients [1]. Aspergillosis of the respiratory system, as well as that of the soft tissues and the skin, due to inhaled spores with infrequent involvement of the gastrointestinal tract, has been well documented [1,3]. Osteomyelitis caused by Aspergillus spp. represents a rare and severe opportunistic infection. Most of such cases involve vertebras, presenting as spondylodiscitis [2]. These infections, particularly in children, may occur through contiguous spread, usually from a pulmonary or sinus infection or from the overlying skin. In older patients, the fungus may also spread hematogenously. However, it is of note that growth of Aspergillus species in blood cultures is extremely rare [3].

The most pathogenic species among Aspergilli is A. fumigatus, while twenty other species may cause infection $[2,3]$. The most common ones are $A$. flavus, $A$. terreus, $A$. nidulans, and A. niger [3]. However, an increase in the number of "cryptic" Aspergillus species has been identified, such as A. lentulus N. pseudofischeri, A. udagawae, A. viridinutans, A. fumigatiaffinis, and A. novofumigatus of the Fumigati section; A. alliaceus of the Flavi section; A. carneus and A. alabamensis of the Terrei section; A. tubingensis, A. awamori, and A. acidus of the Nigri section; A. sydowii of the Versicolores section; A. westerdijkiae and A. persii of the Circumdati section; and A. calidoustus, A. insuetus, and A. keveii of the Usti section. Nevertheless, the clinical context has been detailed only for a very limited number of these strains and information regarding AFT effectiveness is even more scarce [4].

This type of osteoarticular infection is not well understood [2-6]. Diagnosis and management of osseous invasive aspergillosis represent a real challenge. The rarity and diversity of the disease's presentation, often lacking an obvious host response to the infection, especially in patients with severe immune deficiencies, make the clinical diagnosis extremely difficult $[1,7]$. Firm diagnosis, achieved by cultures and/or histopathology, following direct sampling and proper therapy are of paramount importance. All patients require causative antifungal treatment (AFT) and many of them require additional surgical intervention. Surgical debridement is considered the gold-standard of chronic bacterial osteomyelitis management. Debridement of fungal osteomyelitis may also be important and involves the removal of sinus tracts. However, it has been a subject of debate, as some Aspergillus osteomyelitis cases that received successful medical treatment did not require surgery $[1,2,7]$. There are scarce data and limited research has been conducted on surgical management of this infection. Hence, official guidelines on when surgical intervention is necessary do not exist.

A. fumigatus is the most common etiologic agent of Aspergillus osteomyelitis, being responsible for approximately $80 \%$ of these cases. Nevertheless, A. flavus and A. terreus may also cause such infections [4].

Few Aspergillus osteomyelitis cases in the appendicular skeleton may be found in the literature. Therefore, a consensus on diagnostic criteria and the most effective medical management is based on limited data.

The present study is a review of all published cases of Aspergillus osteomyelitis in an effort to describe epidemiology, patients' characteristics, as well as medical and surgical treatment options and their effectiveness.

\section{Methods}

A thorough electronic search of the PubMed and MEDLINE databases was performed to locate all existing articles related to Aspergillus osteomyelitis cases from January 2003 to October 2021. Alone and/or in combination, the terms "Aspergillus osteomyelitis", "fungal osteomyelitis", "Aspergillus osseous infection", "Aspergillus fumigatus osteomyelitis", "Aspergillus bone infection", and "fungal skeleton infection" were searched. In addition, terms including each Aspergillus species (e.g., "Aspergillus terreus osteomyelitis", 
"Aspergillus flavus osteomyelitis", and so on) were also searched. Following the identification of these reports, individual references from each publication were further reviewed for locating additional cases.

The review was limited to papers published in English and in peer-reviewed journals. Expert opinions; book chapters; studies on animals, on cadavers or in vitro investigations; as well as abstracts of scientific meetings were excluded. This review is also limited to cases published after 2003, as voriconazole, which has been as proven the drug of choice against Aspergillus spp. and changed the therapeutic results, was introduced that year. Furthermore, vertebral as well as skull infections were excluded.

The data extracted from these studies included age, gender, location of the osseous infection, responsible Aspergillus species, other site of Aspergillosis, co-infection with bacterial species, C-reactive protein (CRP) and erythrocyte sedimentation rate (ESR) at initial presentation, presence of immunosuppressive condition, duration and type of AFT, and type of surgical intervention. Furthermore, the results of medical and surgical treatment, along with the follow-up of each case, were evaluated. Treatment was considered successful if all signs and symptoms of the infection disappeared and no recurrence was observed during the follow-up period.

Data were recorded and analyzed using Microsoft Excel 2019 (Microsoft Corporation, Redmond, WA, USA).

\section{Results}

A total of 63 patients ( 46 males; $73 \%$ ), with a mean age of 37.9 years [standard deviation $(\mathrm{SD})=25.3]$, suffering osteomyelitis due to Aspergillus spp. were identified during the study period [1,7-57].

A total of 68 osseous infections were recorded because, in five patients, two sites of infection were observed (cases 7, 14, 15, 16, and 42 in Table 1). Regarding the site of infection, the rib cage represented the most commonly affected region ( 25 cases; $36.8 \%$ ); followed by the sternum $(13 ; 19.1 \%)$; the tibia $(7 ; 10.3 \%)$; the femur $(5 ; 7.4 \%)$; the ankle and the foot ( 4 each; $5.9 \%$ ); the humerus ( 3 each; $4.4 \%$ ); the ilium and the scapula ( 2 each; $2.9 \%$ ); and the patella, the wrist, and the fibula (1 each; $1.5 \%$ ).

Table 1. Main characteristics of the published osteomyelitis cases due to Aspergillus spp. Year of publication, patient's demographics, responsible Aspergillus spp., site of infection, immunosuppressive condition and/or medications, other site of Aspergillosis, and symptoms. M: male, F: female, CGD: chronic granulomatous disease, TBC: tuberculosis, LT: lung transplant, RT: renal transplant, IST: immunosuppressive treatment, DM: diabetes mellitus, HT: heart transplant, LSI: local signs of inflammation.

\begin{tabular}{|c|c|c|c|c|c|c|c|c|}
\hline Case No & Year & Reference & $\begin{array}{l}\text { Gender/ } \\
\text { Age }\end{array}$ & $\begin{array}{l}\text { Aspergillus } \\
\text { Species }\end{array}$ & Location & $\begin{array}{c}\text { Previous } \\
\text { Surgery or } \\
\text { Trauma of the } \\
\text { Affected Region }\end{array}$ & $\begin{array}{l}\text { Immunosuppressive } \\
\text { Conditions and/or } \\
\text { Medications }\end{array}$ & Symptoms \\
\hline 1. & 2003 & [8] & $\mathrm{M} / 16$ & A nidulans & femur & - & CGD & Pain, pyrexia \\
\hline 2. & 2003 & [9] & $\mathrm{M} / 12$ & spp. & ilium & Yes & CGD & $\begin{array}{l}\text { Pain, restriction of } \\
\text { ROM, pyrexia }\end{array}$ \\
\hline 3. & 2003 & [10] & $\mathrm{M} / 17$ & A fumigatus & patella & - & $\begin{array}{l}\text { TBC, antituberculosis } \\
\text { therapy }\end{array}$ & $\begin{array}{c}\text { Pyrexia, } \\
\text { lymphadenopathy }\end{array}$ \\
\hline 4. & 2003 & [11] & $\mathrm{F} / 13$ & spp. & ilium & - & Leukemia, chemotherapy & Pyrexia, pain \\
\hline 5. & 2003 & [12] & $\mathrm{F} / 8$ & A fumigatus & rib cage & - & CGD & - \\
\hline 6. & 2004 & [13] & $\mathrm{M} / 48$ & A fumigatus & rib cage & - & $\begin{array}{l}\text { Heroin abuse, methadone } \\
\text { replacement }\end{array}$ & Pain, weight loss \\
\hline 7. & 2004 & [14] & $\mathrm{M} / 64$ & A fumigatus & foot, ankle & - & Bilateral LT recipient, IST & LSI, pain, pyrexia \\
\hline 8. & 2004 & [15] & $\mathrm{M} / 74$ & A flavus & sternum & - & Renal failure & $\begin{array}{l}\text { Fatigue, malaise, } \\
\text { pyrexia LSI, } \\
\text { sterno-cutaneous } \\
\text { fistula }\end{array}$ \\
\hline
\end{tabular}


Table 1. Cont.

\begin{tabular}{|c|c|c|c|c|c|c|c|c|}
\hline Case No & Year & Reference & $\begin{array}{l}\text { Gender/ } \\
\text { Age }\end{array}$ & $\begin{array}{l}\text { Aspergillus } \\
\text { Species }\end{array}$ & Location & $\begin{array}{c}\text { Previous } \\
\text { Surgery or } \\
\text { Trauma of the } \\
\text { Affected Region }\end{array}$ & $\begin{array}{l}\text { Immunosuppressive } \\
\text { Conditions and/or } \\
\text { Medications }\end{array}$ & Symptoms \\
\hline 9. & 2004 & [15] & $\mathrm{M} / 63$ & A flavus & sternum & - & $\begin{array}{l}\text { Chronic obstructive } \\
\text { pulmonary disease }\end{array}$ & $\begin{array}{c}\text { Fatigue, malaise, } \\
\text { pyrexia LSI, } \\
\text { sterno-cutaneous } \\
\text { fistula }\end{array}$ \\
\hline 10. & 2004 & [15] & $\mathrm{M} / 56$ & A flavus & sternum & - & DM, asthma & $\begin{array}{c}\text { Fatigue, malaise, } \\
\text { pyrexia LSI, } \\
\text { sterno-cutaneous } \\
\text { fistula }\end{array}$ \\
\hline 11. & 2005 & [16] & $\mathrm{M} / 65$ & spp. & rib cage & - & $\begin{array}{c}\text { Heavy smoker, alcoholism, } \\
\text { TBC }\end{array}$ & Pain, weight loss \\
\hline 12. & 2005 & [17] & $\mathrm{M} / 40$ & A versicolor & foot & - & $\begin{array}{l}\text { Acute lymphoblastic } \\
\text { leukemia, chemotherapy, } \\
\text { renal failure }\end{array}$ & Pain, LSI, pyrexia \\
\hline 13. & 2005 & [18] & $\mathrm{M} / 62$ & A fumigatus & rib cage & Yes & $\begin{array}{l}\text { DM, chronic obstructive } \\
\text { pulmonary disease }\end{array}$ & Pain, LSI \\
\hline 14. & 2005 & [19] & $\mathrm{M} / 5$ & A fumigatus & $\begin{array}{l}\text { rib cage and } \\
\text { wrist }\end{array}$ & - & - & LSI, pain \\
\hline 15. & 2005 & [20] & $\mathrm{M} / 43$ & A fumigatus & $\begin{array}{l}\text { femur and } \\
\text { fibula }\end{array}$ & Yes & - & - \\
\hline 16. & 2005 & [20] & $\mathrm{M} / 31$ & A fumigatus & rib cage & - & AIDS & - \\
\hline 17. & 2005 & [20] & $\mathrm{M} / 4$ & A fumigatus & rib cage & - & CGD & - \\
\hline 18. & 2005 & [20] & $\mathrm{F} / 4$ & A nidulans & $\begin{array}{l}\text { sternum, } \\
\text { femur }\end{array}$ & - & CGD & - \\
\hline 19. & 2005 & {$[20]$} & $\mathrm{M} / 14$ & A fumigatus & rib cage & - & CGD & - \\
\hline 20. & 2005 & [20] & $\mathrm{M} / 9$ & A fumigatus & rib cage & - & Acute lymphocytic leukemia & - \\
\hline 21. & 2007 & [21] & $\mathrm{M} / 8$ & A fumigatus & rib cage & - & CGD & - \\
\hline 22. & 2007 & {$[22]$} & $\mathrm{M} / 39$ & A fumigatus & sternum & - & TBC, AIDS, hepatitis B & Pain \\
\hline 23. & 2007 & [23] & $\mathrm{F} / 29$ & A terreus & sternum & - & DM, RT recipient, IST & LSI \\
\hline 24. & 2007 & [24] & $\mathrm{M} / 34$ & A fumigatus & $\begin{array}{l}\text { ankle (talar } \\
\text { bone) }\end{array}$ & - & $\begin{array}{c}\text { RT recipient, hepatitis C, IST, } \\
\text { DM, TBC }\end{array}$ & $\begin{array}{l}\text { LSI, pain, } \\
\text { restriction of ROM }\end{array}$ \\
\hline 25. & 2008 & [25] & $\mathrm{M} / 21$ & A nidulans & rib cage & - & CGD & LSI \\
\hline 26. & 2008 & [26] & $\mathrm{M} / 60$ & A fumigatus & sternum & - & DM, smoker, heart failure & $\begin{array}{c}\text { LSI, } \\
\text { sterno-cutaneous } \\
\text { fistula }\end{array}$ \\
\hline 27. & 2008 & {$[27]$} & $-/ 14$ & spp. & rib cage & - & CGD & - \\
\hline 28. & 2008 & [28] & $\mathrm{M} / 69$ & A flavus & tibia & Yes & $\mathrm{DM}$ & LSI, ulcer \\
\hline 29. & 2009 & [29] & $\mathrm{M} / 70$ & A flavus & rib cage & - & - & Pain \\
\hline 30. & 2009 & [30] & $\mathrm{M} / 18$ & A fumigatus & foot & - & CGD & - \\
\hline 31. & 2009 & [31] & $\mathrm{F} / 60$ & A fumigatus & humerus & - & $\begin{array}{l}\mathrm{RT} \text { and pancreas transplant } \\
\text { recipient, IST }\end{array}$ & - \\
\hline 32. & 2009 & [31] & $\mathrm{F} / 62$ & A fumigatus & rib cage & - & $\begin{array}{l}\text { Lung cancer (remission), } \\
\text { chemotherapy }\end{array}$ & - \\
\hline 33. & 2009 & [31] & $\mathrm{M} / 63$ & A fumigatus & sternum & - & - & - \\
\hline 34. & 2009 & [31] & $\mathrm{F} / 79$ & A fumigatus & sternum & - & - & - \\
\hline 35. & 2010 & [32] & $\mathrm{M} / 4$ & spp. & femur & - & $\begin{array}{c}\text { Acute lymphatic } \\
\text { leukemia, } \\
\text { chemotherapy }\end{array}$ & Pyrexia, pain \\
\hline 36. & 2011 & [33] & $\mathrm{F} / 79$ & A fumigatus & femur & - & $\begin{array}{l}\text { Lymphocytic leukemia, } \\
\text { chemotherapy }\end{array}$ & Pain \\
\hline 37. & 2011 & [34] & $\mathrm{F} / 13$ & A flavus & tibia & Yes & - & $\begin{array}{c}\text { Pain, restriction of } \\
\text { ROM }\end{array}$ \\
\hline 38. & 2011 & [35] & $\mathrm{M} / 37$ & spp. & humerus & - & RT recipient, IST, hepatitis C & Pain, weight loss \\
\hline 39. & 2012 & [36] & $\mathrm{F} / 2.5$ & spp. & tibia & - & CGD & LSI, pyrexia \\
\hline 40. & 2012 & [37] & $\mathrm{M} / 13$ & A nidulans & tibia & & CGD & - \\
\hline 41. & 2012 & [38] & $\mathrm{F} / 72$ & A fumigatus & humerus & Yes & DM, heart failure & Pain \\
\hline 42. & 2013 & [39] & $\mathrm{M} / 69$ & A fumigatus & $\begin{array}{l}\text { sternum and } \\
\text { rib cage }\end{array}$ & - & - & LSI \\
\hline
\end{tabular}


Table 1. Cont.

\begin{tabular}{|c|c|c|c|c|c|c|c|c|}
\hline Case No & Year & Reference & $\begin{array}{l}\text { Gender/ } \\
\text { Age }\end{array}$ & $\begin{array}{l}\text { Aspergillus } \\
\text { Species }\end{array}$ & Location & $\begin{array}{c}\text { Previous } \\
\text { Surgery or } \\
\text { Trauma of the } \\
\text { Affected Region }\end{array}$ & $\begin{array}{l}\text { Immunosuppressive } \\
\text { Conditions and/or } \\
\text { Medications }\end{array}$ & Symptoms \\
\hline 43. & 2013 & [40] & $\mathrm{M} / 54$ & A flavus & rib cage & - & $\begin{array}{l}\text { DM, renal failure, } \\
\text { heart failure }\end{array}$ & Pain, pyrexia \\
\hline 44. & 2013 & {$[41]$} & $\mathrm{F} / 45$ & A fumigatus & rib cage & - & $\mathrm{DM}$ & Pain, weight loss \\
\hline 45. & 2013 & [42] & $\mathrm{F} / 30$ & spp. & foot & Yes & - & LSI \\
\hline 46. & 2014 & {$[43]$} & $\mathrm{M} / 28$ & A flavus & tibia & - & - & LSI, pain \\
\hline 47. & 2015 & [44] & $\mathrm{F} / 26$ & & tibia & Yes & - & $\begin{array}{l}\text { LSI, pain, drainage, } \\
\text { restriction of ROM }\end{array}$ \\
\hline 48. & 2015 & [45] & $\mathrm{M} / 18$ & A flavus & rib cage & - & $\begin{array}{c}\text { Acute lymphoblastic } \\
\text { leukemia B }\end{array}$ & - \\
\hline 49. & 2015 & [46] & $\mathrm{M} / 68$ & A flavus & $\begin{array}{c}\text { ankle } \\
\text { (calcaneus) }\end{array}$ & - & $\mathrm{DM}$ & Pyrexia, LSI, ulcer \\
\hline 50. & 2016 & [47] & $\mathrm{M} / 4$ & spp. & tibia & - & CGD & Pain \\
\hline 51. & 2016 & {$[48]$} & $\mathrm{F} / 4.5$ & A fumigatus & rib cage & - & CGD, TBC & Pyrexia, pain \\
\hline 52. & 2017 & [49] & $\mathrm{M} / 51$ & A flavus & rib cage & - & - & Pain, pyrexia \\
\hline 53. & 2017 & [49] & $\mathrm{M} / 40$ & A flavus & rib cage & Yes & - & Pain, pyrexia \\
\hline 54. & 2018 & {$[50]$} & $\mathrm{M} / 7$ & fumigatus & $\begin{array}{l}\text { ankle (talar } \\
\text { bone) }\end{array}$ & - & CGD & Pain, drainage \\
\hline 55. & 2018 & [51] & $\mathrm{M} / 70$ & fumigatus & sternum & - & $\begin{array}{l}\text { Chronic obstructive } \\
\text { pulmonary disease }\end{array}$ & Pain \\
\hline 56. & 2018 & [7] & $\mathrm{M} / 9$ & spp. & scapula & - & CGD & Pain, pyrexia \\
\hline 57. & 2019 & [52] & $\mathrm{F} / 61$ & A flavus & rib cage & - & $\begin{array}{l}\text { Smoker, breast cancer, } \\
\text { radiotherapy, chronic } \\
\text { obstructive pulmonary } \\
\text { disease, lymphoma, } \\
\text { chemotherapy }\end{array}$ & Pain \\
\hline 58. & 2020 & {$[53]$} & $\mathrm{M} / 65$ & A fumigatus & sternum & Yes & HT recipient, IST & LSI \\
\hline 59. & 2020 & [54] & $\mathrm{M} / 37$ & A fumigatus & rib cage & - & - & LSI \\
\hline 60. & 2020 & [55] & $\mathrm{M} / 10$ & A nidulans & rib cage & - & CGD & $\begin{array}{c}\text { Pain, night sweets, } \\
\text { lethargy, coryza, } \\
\text { weakness }\end{array}$ \\
\hline 61. & 2020 & [1] & $\mathrm{M} / 52$ & A fumigatus & scapula & - & HT recipient, IST & Pain \\
\hline 62. & 2020 & [56] & $\mathrm{F} / 65$ & spp. & sternum & - & DM, heart failure & LSI \\
\hline 63. & 2021 & [57] & $\mathrm{M} / 77$ & A fumigatus & rib cage & - & $\begin{array}{l}\text { Rheumatoid arthritis, } \\
\text { chronic obstructive } \\
\text { pulmonary disease, DM }\end{array}$ & Pain, drainage \\
\hline
\end{tabular}

Mean CRP and ESR upon initial presentation were $49.6 \mathrm{mg} / \mathrm{L}(\mathrm{SD}=72.9)$ and $72.4 \mathrm{~mm} / \mathrm{h}(\mathrm{SD}=34.7)$, respectively. Another site of Aspergillus infection was reported in 17 patients $(27 \%)$. The mean follow-up was found to be 12.2 months $(\mathrm{SD}=11.6)$. Furthermore, 48 patients $(76.2 \%)$ were immunocompromised according to the available information from each report. The majority of these patients suffered from chronic granulomatous disease (17 cases; $35.4 \%$ ), followed by patients with diabetes mellitus (12 cases; $25 \%$ ), organ transplant recipients under immunosuppressive therapy ( 7 cases; $14.6 \%)$, and patients receiving chemotherapy ( 6 cases; $12.5 \%)$. Additionally, it is of note that 10 patients $(15.9 \%)$ had suffered trauma and/or underwent surgery involving the infected area.

Details on patients' symptomology are thoroughly presented in Table 1. Pain represented the main complaint in most cases $(32 ; 50.8 \%)$, followed by local symptoms of inflammation in $21(33.3 \%)$, pyrexia in $17(27 \%)$, and weight loss in $4(6.3 \%)$.

Regarding imaging methods indicating osseous infection, computer tomography (CT) was performed in 27 patients $(42.9 \%)$, followed by plain X-ray in $26(41.3 \%)$ and magnetic resonance imaging (MRI) in 22 (34.9\%). In 13 cases (cases 5, 21, 23, 27, 29-34, 40, 43, and 48 in Table 1), no imaging was reported.

All osteomyelitis cases due to Aspergillus spp. were diagnosed through cultures and/or histopathology. Galactomannan antigen test was additionally used in seven cases (cases 
$1,22,23,24,25,36$, and 55 in Table 1), while polymerase chain reaction (PCR) was used in four cases (cases 1, 49, 57, and 59 in Table 1). Furthermore, in three cases (cases 55, 58, and 59 in Table 1), beta-D-glucan testing was additionally performed.

A total of 63 Aspergillus spp. strains were isolated. The most commonly isolated was A. fumigatus (31 strains; $49.2 \%)$, followed by $A$. flavus (13; 20.6\%), A. nidulans ( $5 ; 7.9 \%)$, and $A$. versicolor and $A$. terreus (1 each; $1.6 \%)$. Additionally, $12(19 \%)$ isolates were not further characterized.

Medical management, as well as the infection's outcome of the reported cases, are highlighted in Table 2. Regarding AFT, 28 cases (44.4\%) were treated with a single antifungal drug, while 18 cases $(28.6 \%)$ were treated with two, either simultaneously or consecutively, and 15 cases $(23.8 \%)$ were treated with more than two antifungal agents. Information regarding the specific antifungal drug was not reported in three cases (4.8\%) (cases 35, 50, and 54 in Table 2). The mean AFT duration was 5.3 months $(\mathrm{SD}=4.9)$.

Table 2. Therapeutic management of osteomyelitis due to Aspergillus spp. Antifungal treatment (AFT), duration of AFT, and infection's outcome are presented. ${ }^{*}$ ): death as a result of infection.

\begin{tabular}{|c|c|c|c|c|}
\hline Case \# & Reference & AFT & Total Duration of AFT (Months) & Infection's Outcome \\
\hline 1. & [8] & $\begin{array}{l}\text { Amphotericin B, } \\
\text { itraconazole }\end{array}$ & undefined long-duration & Success \\
\hline 2. & [9] & Amphotericin B & 1.5 & Success \\
\hline 3. & [10] & $\begin{array}{l}\text { Amphotericin B, } \\
\text { itraconazole }\end{array}$ & 6 & Success \\
\hline 4. & [11] & Voriconazole & 6 & Success \\
\hline 5. & [12] & $\begin{array}{l}\text { Amphotericin B, } \\
\text { itraconazole }\end{array}$ & 3.5 & Failure * \\
\hline 6. & [13] & Itraconazole & 18 & Success \\
\hline 7. & [14] & $\begin{array}{l}\text { Itraconazole, amphotericin } \mathrm{B}, \\
\text { posaconazole }\end{array}$ & 12 & Success \\
\hline 8. & [15] & $\begin{array}{l}\text { Amphotericin B, } \\
\text { itraconazole }\end{array}$ & 1 & Success \\
\hline 9. & [15] & $\begin{array}{l}\text { Amphotericin B, } \\
\text { itraconazole }\end{array}$ & 3 & Failure * \\
\hline 10. & [15] & $\begin{array}{l}\text { Amphotericin B, } \\
\text { itraconazole }\end{array}$ & 3 & Success \\
\hline 11. & [16] & Amphotericin B & - & Failure * \\
\hline 12. & [17] & $\begin{array}{l}\text { Amphotericin B, fluconazole, } \\
\text { itraconazole }\end{array}$ & 2 & Failure * \\
\hline 13. & [18] & Itraconazole & 6 & Success \\
\hline 14. & [19] & Amphotericin B, itraconazole & undefined long-duration & Success \\
\hline 15. & [20] & $\begin{array}{c}\text { Amphotericin B, } \\
\text { itraconazole, voriconazole }\end{array}$ & 3 & Success \\
\hline 16. & {$[20]$} & $\begin{array}{l}\text { Amphotericin B, 5-flucytosine, } \\
\text { itraconazole, voriconazole }\end{array}$ & 3 & Success \\
\hline 17. & {$[20]$} & Amphotericin B, voriconazole & 6 & Success \\
\hline 18. & [20] & $\begin{array}{l}\text { Amphotericin B, itraconazole, } \\
\text { 5-flucytosine, voriconazole }\end{array}$ & 6 & Success \\
\hline 19. & [20] & $\begin{array}{l}\text { Amphotericin B, itraconazole, } \\
\text { voriconazole }\end{array}$ & 1 & Failure * \\
\hline 20. & [20] & Amphotericin B, voriconazole & 2.5 & Success \\
\hline
\end{tabular}


Table 2. Cont.

\begin{tabular}{|c|c|c|c|c|}
\hline Case \# & Reference & AFT & Total Duration of AFT (Months) & Infection's Outcome \\
\hline 21. & [21] & Amphotericin B, itraconazole & 1 & Success \\
\hline 22. & [22] & Voriconazole & - & Success \\
\hline 23. & [23] & $\begin{array}{l}\text { Amphotericin B, itraconazole, } \\
\text { caspofungin, voriconazole }\end{array}$ & 2 & Success \\
\hline 24. & [24] & Amphotericin B & 1.5 & Success \\
\hline 25. & [25] & $\begin{array}{l}\text { Amphotericin B, caspofungin, } \\
\text { voriconazole }\end{array}$ & 5 & Failure * \\
\hline 26. & [26] & Voriconazole & 0.5 & Success \\
\hline 27. & [27] & $\begin{array}{l}\text { Amphotericin B, } \\
\text { itraconazole }\end{array}$ & - & Failure * \\
\hline 28. & [28] & Voriconazole & 7 & Success \\
\hline 29. & [29] & Voriconazole & 6 & Success \\
\hline 30. & {$[30]$} & $\begin{array}{l}\text { Voriconazole, caspofungin, } \\
\text { posaconazole, itraconazole }\end{array}$ & 12 & Success \\
\hline 31. & {$[31]$} & Voriconazole, posaconazole, micafungin & 2.5 & Failure * \\
\hline 32. & [31] & Voriconazole & 0.5 & Success \\
\hline 33. & [31] & Fluconazole & 0.5 & Failure * \\
\hline 34. & [31] & Voriconazole & 1 & Success \\
\hline 35. & [32] & Two antifungal agents & 6 & Success \\
\hline 36. & {$[33]$} & Amphotericin B, voriconazole & - & Success \\
\hline 37. & {$[34]$} & Voriconazole, caspofungin & 12 & Success \\
\hline 38. & {$[35]$} & Voriconazole & 2 & Success \\
\hline 39. & {$[36]$} & Voriconazole & 3 & Success \\
\hline 40. & {$[37]$} & Voriconazole & 1 & Success \\
\hline 41. & [38] & Voriconazole & 18 & Success \\
\hline 42. & {$[39]$} & Voriconazole & 6 & Success \\
\hline 43. & {$[40]$} & Amphotericin B, itraconazole & 6 & Success \\
\hline 44. & {$[41]$} & Voriconazole & - & Success \\
\hline 45. & {$[42]$} & Amphotericin B & 1.5 & Success \\
\hline 46. & {$[43]$} & Amphotericin B, voriconazole & 3 & Success \\
\hline 47. & {$[44]$} & Tioconazole & 0.75 & Success \\
\hline 48. & {$[45]$} & Caspofungin, voriconazole & & Success \\
\hline 49. & {$[46]$} & Amphotericin B, voriconazole & 4 & Success \\
\hline 50. & [47] & - & - & - \\
\hline 51. & [48] & $\begin{array}{c}\text { Amphotericin B, itraconazole, } \\
\text { 5-fluorocytosine }\end{array}$ & 2 & Failure * \\
\hline 52. & [49] & Voriconazole & 4 & Success \\
\hline 53. & [49] & Voriconazole & 3 & Success \\
\hline 54. & {$[50]$} & - & - & Failure * \\
\hline 55. & [51] & Voriconazole & 6 & Success \\
\hline
\end{tabular}


Table 2. Cont.

\begin{tabular}{ccccc}
\hline Case \# & Reference & AFT & Total Duration of AFT (Months) & Infection's Outcome \\
\hline 56. & {$[7]$} & Voriconazole & 3.5 & Success \\
\hline 57. & {$[52]$} & Voriconazole & 8.6 & Success \\
\hline 58. & {$[53]$} & $\begin{array}{c}\text { Amphotericin B, voriconazole, } \\
\text { isavuconazole }\end{array}$ & 10 & Success \\
\hline 59. & {$[54]$} & $\begin{array}{c}\text { Amphotericin B, voriconazole, } \\
\text { posaconazole }\end{array}$ & 9 & Success \\
\hline 60. & {$[55]$} & Amphotericin B, voriconazole, \\
caspofungin & Failure * & Success \\
\hline 61. & {$[1]$} & Voriconazole & 24 & - \\
\hline 62. & {$[56]$} & $\begin{array}{c}\text { Amphotericin B, micafungin, } \\
\text { voriconazole, isavuconazole, }\end{array}$ & 4 & Failure * \\
\hline 63. & {$[57]$} & Voriconazole & 42 & \\
\hline
\end{tabular}

Voriconazole was the preferred antifungal, used in 39 cases [(61.9\%), in $20(51.3 \%)$ as monotherapy]; followed by amphotericin B in 32 [(50.8\%), in $4(12.5 \%)$ as monotherapy]; itraconazole in 21 [(33.3\%), in $2(9.5 \%)$ as monotherapy]; caspofungin in $6[(9.5 \%)$, none as monotherapy]; posaconazole in 4 [(6.3\%), none as monotherapy]; flucytosine in 3 [( $4.8 \%)$, none as monotherapy]; fluconazole, micafungin, and isavuconazole in 2 [( $3.2 \%)$, fluconazole in 1 case $(50 \%)$ as monotherapy, while the other drugs were given in combination with additional antifungals]; and tioconazole in 1 [(1.6\%), as monotherapy].

The infection's outcome was successful in 48 cases $(76.2 \%)$, while the mortality rate attributed to the infection and/or its complications was found to be $20.6 \%$.

Surgical debridement was additionally performed in 40 cases $(63.5 \%)$. The infection's outcome in these cases was successful in 31 cases $(77.5 \%)$, while the mortality rate was $22.5 \%$.

\section{Discussion}

Fungi of the Aspergillus species may lead to severe infections in human hosts, including a broad range of clinical presentations, such as aspergilloma (or fungus ball in the lung), severe asthma, sinus fungus ball and granulomatous sinusitis, otomycosis, keratitis, endophthalmitis, skin, wound and osteoarticular infections, chronic pulmonary aspergillosis, tracheobronchitis, invasive pulmonary, and disseminated disease $[4,58]$. Invasive Aspergillosis represents an important cause of morbidity and mortality in immunosuppressed hosts. It has been estimated that about 200,000 cases of invasive Aspergillosis occur worldwide per annum. However, this does not account for all actual cases, as a lack of accuracy in the diagnosis or treatment has led to increased mortality that ranges between $20 \%$ and $100 \%[4,58]$.

Osteoarticular Aspergillosis is not a common infection, while, owing to its rarity, it is not well described or understood. Data and information about Aspergillus osteomyelitis are scarce $[34,39,59]$. The present study reviewed all osteomyelitis cases caused by Aspergillus spp., reported from 2003 to 2021 in the literature, scoping to elucidate epidemiology, patient's characteristics, causative Aspergillus spp., as well as the medical and surgical treatment and their effectiveness.

Voriconazole is an antifungal agent introduced in 2003 and represents the treatment of choice for invasive Aspergillosis. This agent changed for the better the results of medical AFT. Alternative AFTs consist of lipid or liposomal formulation of amphotericin B and isavuconazole, while other treatments used as salvage therapies are caspofungin, micafungin, anidulafungin, posaconazole, and itraconazole $[2,60]$. Therefore, the present review is limited to cases published after 2003. 
The present study reviewed 63 cases of osteomyelitis, yielding 63 Aspergillus spp. strains. The present sample was rather young, as the patients' mean age was about 38 years, ranging from to 2.5 to 79 years, while the male gender was highly represented (73\% males). However, it is of note that another site of Aspergillus infection was evident in 17 patients $(27 \%)$. Therefore, several patients did not suffer from an apparent pulmonary or other extra-osseous Aspergillus focus, indicating that isolated Aspergillus osteomyelitis may occur de novo. It is also of note that 10 patients $(15.9 \%)$ had suffered trauma and/or underwent surgery involving the infected area. Hence, it should be kept in mind that non-immunocompromised patients may also be at risk, as previous surgical procedures or trauma may serve as a source of direct inoculation.

In the present review, the majority of affected patients were immunocompromised $(76.2 \%)$. More particular, most of the hosts suffered from chronic granulomatous disease $(35.4 \%)$, followed by patients with diabetes mellitus $(25 \%)$, organ transplant recipients under immunosuppressive therapy (14.6\%), and patients receiving chemotherapy (12.5\%). Regarding immunosuppressive conditions, there has been documented a spectrum of risk for invasive Aspergillosis. More specifically, conditions having high risk include chronic granulomatous disease, lung or hear transplantation, and leukemia under chemotherapy, while conditions with intermediate risk include liver transplantation, small bowel transplantation, myelodysplastic syndrome, and kidney failure. Finally, clinical entities with low risk include multiple myeloma, chronic obstructive pulmonary disease, non-Hodgkin's lymphoma, solid tumor, AIDS, and diabetes mellitus [61]. It is believed that approximately 10 million patients with an impaired immune system per year are considered at risk to develop invasive aspergillosis [4].

It should be highlighted that almost $15 \%$ of the study's immunocompromised patients were organ transplant recipients. In these cases, it is important, if such an infection is presumed, to reduce the immunosuppressive treatment, as the degree of immunosuppression strongly influences the outcome of invasive aspergillosis. Furthermore, it should be taken into consideration that voriconazole, the antifungal drug of choice, interferes with the P450 cytochrome oxidase $[1,14,35]$. Hence, this agent may alter the activity and the levels of some immunosuppressive drugs $[14,35]$.

The onset of fungal infections is often insidious with non-specific symptoms, thus diagnosis is often a challenge [35,62-64]. As highlighted in Table 1, pain, local signs of infection, and fever represent the main symptoms of osteomyelitis caused by Aspergillus spp. Such symptoms are common clinical indicators for most osseous infections. Additionally, no other specific clinical manifestation exists that could consistently differentiate between bacterial and Aspergillus osteomyelitis. Therefore, the laboratory firm diagnosis is of utmost important for the identification of the causative microorganism. Early recognition of Aspergillus osteomyelitis plays a major role in the infection's outcome.

Systemic inflammatory markers, such as CRP and ESR, which represent markers mainly used in every-day clinical practice, may be minimally elevated or even normal in cases of fungal osteomyelitis $[4,61,62]$. Hence, a detailed medical history, focused on potential immunosuppressive conditions and/or medications, as well as a thorough physical examination, are very important. In the present study, mean CRP and ESR upon initial presentation were found to be $49.6 \mathrm{mg} / \mathrm{L}$ and $72.4 \mathrm{~mm} / \mathrm{h}$, respectively.

Imaging techniques also play an important role facilitating the diagnosis. In the present study, CT was performed in most cases (42.9\%), followed by plain X-ray (41.3\%) and MRI (34.9\%). In plain X-rays, suspicious signs for osteomyelitis include cortical erosion, permeative marrow lucency and periosteal reaction or sclerosis, and cortical thickening. CT scans demonstrate subtle cortical erosions earlier than radiographs and reveal sequestra [65]. MRIs reveal typical findings of osteomyelitis, including edema and enhancement of bone marrow, along with the replacement of bright fatty marrow signal on T1 weighted images with signal closer to the muscle intensity. T2 marrow hyperintensity and enhancement, as well as T2 hyperintense periosteal edema, may be reactive to adjacent 
soft tissue infection. Hence, T1 marrow replacement is the most specific sign of marrow infection [65].

The present review has revealed that the most common site of osteomyelitis due to Aspergillus spp. is the rib cage (36.8\%), followed by the sternum (19.1\%), the tibia (10.3\%), and the femur $(7.4 \%)$. Other sites, such as the ankle, the foot, the humerus, the ilium, the scapula, the patella, the wrist, and the fibula, were also identified, but not highly represented.

Aspergilli are relative common food and soil contaminants, while their spores are ubiquitous $[4,58,62]$. The most frequent species involved in human infection is Aspergillus fumigatus $[2,58,62]$. Although $A$. fumigatus is the most common etiologic agent, being responsible for approximately $80 \%$ of the cases of Aspergillosis, A. flavus and A. terreus may also cause such infections. In the present study, $A$. fumigatus was the most common isolated one $(49.2 \%)$, while A. flavus $(20.3 \%)$, A. nidulans $(7.9 \%)$, and A. versicolor and A. terreus $(1.6 \%$ each) followed.

In all cases of the present study, the causative Aspergillus strain was isolated from clinical specimens and was identified with cultures and/or histopathology, while galactomannan antigen test was additionally used in seven patients (11.1\%) and PCR in four patients $(6.3 \%)$. Additionally, in three patients (4.8\%), beta-D-glucan testing was additionally performed.

Conventional identification methods of Aspergillus spp. include macroscopic as well as microscopic morphological characteristics [61,62]. Furthermore, additional methods include serum beta-D-glucan, which is a component of the fungal cell wall, PCR testing, and galactomannan antigen test. Serum beta-D-glucan has proved to be helpful. However, this marker does not possess specificity for Aspergillus spp., thus its presence may indicate other invasive fungal infections as well. Galactomannan antigen test provides, mainly, prognostic information with declining values related to effective AFT. PCR testing has not been widely adopted owing to a lack of standardization $[4,61]$.

In all the present cases, the mold was isolated with conventional techniques, while additional methods were also performed in a few cases. Regarding cultures, Aspergillus spp. grow generally well on standard media. However, the use of fungal-specific media may increase this mold's isolation. In some cases, it represents a real challenge to determine the clinical significance of positive cultures from nonsterile sites, while medical history, risk factors, and imaging findings may be extremely helpful. It must be taken into account, though, that AFT may decrease the cultures' yield. Regarding histopathology, direct visualization of the branching septate hyphae on microscopic examination along with recovery of the organism in cultures provide a definite diagnosis $[2,4,61]$.

A major issue in this infection's management is the development of resistance of Aspergillus to antifungal agents, including the azole compounds $[6,62,66,67]$. Owing to overuse of pesticides containing azoles, mainly in the Netherlands and England, an increased azole resistance has been observed in these countries. Nevertheless, in other countries, this has not yet become an issue [61]. Hence, it is of paramount importance to identify the mold and its susceptibilities and to attain accurate MIC values. The EUCAST has standardized antifungal clinical breakpoints for several methods measuring MICs. However, it should be noted that the immune condition of the host is the most critical issue regarding the success of antifungal medical management $[68,69]$.

The present study has shown that, in the majority of cases, voriconazole was the preferred drug, used in 39 cases $(61.9 \%)$, either as monotherapy or in combination with another antifungal, while amphotericin B was used in 32 cases (50.8\%).

Triazoles and amphotericin $B$ represent the antifungal drugs proposed for the management of invasive Aspergillosis, possessing cidal action, while echinocandins have also been used, possessing only static effects. The triazoles exert their antifungal effects by inhibiting the cytochrome P450 (CYP)-dependent 14-a-demethylase, blocking the conversion of lanosterol to ergosterol. Voriconazole, an agent that possesses the features of azole compounds, such as moderate hepatotoxicity and minor nephrotoxicity, as compared with 
all amphotericin formulations, was introduced and approved in 2003 as the antifungal regimen of choice against invasive aspergillosis [60,67]. Voriconazole is a triazole with a broad spectrum of antifungal activity. Its efficacy against all Aspergilli of clinical importance is this drug's most important characteristic. In particular, this antifungal targets the synthesis of ergosterol biosynthesis by inhibition of the cytochrome P450-dependent enzyme lanosterol 14-alpha-demethylase, resulting eventually in severe cell membrane damages and, consequently, inhibition of the fungal cell growth and replication or death. This drug also inhibits enzymes associated with the function of the P450 cytochrome, regulating the functional respiration chain $[1,62]$. This drug's serum concentrations are significantly influenced by a broad range of other drugs owing to extensive drug to drug interactions. Voriconazole is available in oral and intravenous preparations [60,62]. Its adverse effects include liver function test abnormalities (15\% of patients), gastrointestinal toxicities (nausea, vomiting, diarrhea), as well as skin rashes. There have also been other, less common, complications described, such as hallucinations, visual abnormalities (mostly related to higher serum concentrations, transient, and reversible), and fluorosis or cutaneous malignancy with long-term administration. It is important to closely monitor dosing in adults, as the drug's levels are unpredictable, owing to a variety of factors, including gender, age, liver disease, and potential genetic polymorphisms in CYP2C19 [60,62,67].

Amphotericin B is a very broad-spectrum antifungal agent that has been used for decades. It is a polyene that is fungicidal and seems to act by creating extra-membranous masses that extract ergosterol from lipid bilayers, leading in cell death and resulting in the development of ion channels, associated with destruction of the fungal cells. Its main problem, prohibiting its long-term use, is the drug's nephrotoxicity and the associated electrolyte disturbances [2,70]. The liposomal formulations of amphotericin B are less nephrotoxic [70]. In the present study, exact information about the amphotericin B type was not available in most of the reports. Nevertheless, it is reasonable to assume that amphotericin B lipid or liposomal formulations have been the drugs of choice during the last two decades, because, during this period, the therapeutic use of deoxycholate amphotericin B has been abandoned.

Itraconazole is an azole available for per os and iv administration. Given per os may be problematic, as its absorption is erratic, making its use difficult for severe infections. Adverse effects include nausea, vomiting, hypertriglyceridemia, hypokalemia, and hepatotoxicity [61]. Furthermore, negative inotropic effects have also been reported, thus it should be used with extreme caution in patients suffering heart failure. Itraconazole may be mainly used in noninvasive or chronic forms of aspergillosis, or following intolerance or toxicity to other triazoles $[61,62]$. In the present study, itraconazole was the preferred antifungal drug in 21 cases (33.3\%), while in most cases (90.5\%), it was used in combination with another antifungal drug.

Posaconazole is another triazole with an analogous structure to itraconazole available in per os and iv formulations. It is highly active in vitro against Aspergillus spp. Posaconazole is metabolized in the liver through glucuronidation and has drug to drug interactions involving of the azole compounds. It is mainly used as prophylaxis in severe immunocompromised, high-risk patients undergoing bone marrow transplant with graft versus host disease and in patients with acute myelogenous leukemia and myelodysplastic syndrome [61]. In the present study, posaconazole was used in four patients (6.3\%) in combination with another antifungal agent.

Isavuconazole is effective against Aspergillus spp. It has been compared to voriconazole and was revealed to be noninferior and to have $17 \%$ fewer complications. It is available in oral and intravenous forms, while adverse effects include nausea, vomiting, and diarrhea $[51,60]$. Isavuconazole was used, in the present study, in just two cases $(3.2 \%)$, in combination with another antifungal regimen.

The echinocandins, including caspofungin, anidulafungin, and micafungin, inhibit the synthesis of 1,3-b-D-glucan via the glucan synthase enzyme. All are available in iv formulations. They are generally well tolerated, but they are fungistatic, rather than 
fungicidal [61]. In the present study, echinocandins were used in eight cases (12.7\%), in combination with other antifungal drugs.

Prolonged AFT is crucial for the treatment of these infections. The mean AFT duration in the present study was 5.3 months.

Invasive Aspergillosis, including osteomyelitis, is a severe infection with high mortality, especially in immunosuppressed hosts, despite the use of effective antifungal drugs [61] The severity of this fungal infection is portrayed through the relative high mortality rate (20.6\%). Thus, in many cases, combination therapy is given, although such guidelines do not exist. This also becomes apparent by the findings of the present review, because, in 33 cases $(52.4 \%)$, two or more antifungal agents were used for the eradication of the infection.

Fungal osteomyelitis also demands, in most cases, surgical debridement. In the present review, most patients (63.5\%) underwent debridement. Surgical debridement includes thorough removal of the sequestrum and the sinus tracts.

The present study has some limitations. Not all information from a number of the reviewed cases was available. Hence, dosages, drug serum-levels, MICs, and side effects of the used antifungal drugs, in most cases, were not described. However, this review provides valuable information about epidemiology, symptomatology, diagnosis, medical and surgical management, as well as outcome of cases of osteomyelitis caused by Aspergillus spp.

In conclusion, osteomyelitis caused by Aspergillus spp. represents a severe and, in many cases, life-threatening infection, as it affects mainly, but not exclusively, immunocompromised hosts. This infection demands prompt diagnosis and early multidisciplinary management, because, in addition to medical treatment, most cases require surgical intervention. Although new techniques, such as PCR testing, have been developed, conventional methods including cultures and histopathology remain the main tools of isolating the causative mold. Prolonged AFT, guided by susceptibility tests, along with surgical debridement represent the most effective therapeutic approach. Additionally, in culture negative for bacteria and/or cocci osteomyelitis cases, a high index of suspicion for fungal pathogens should be present, especially in immunocompromised hosts.

Author Contributions: Conceptualization, C.K. and G.S.; methodology, K.A., K.R., I.C.; formal analysis, I.C., C.K., G.V.; investigation, K.A., K.R.; resources, G.V., G.S.; data curation, I.C., K.A.; writing-original draft preparation, C.K., I.C.; writing-review and editing, K.A., G.V., G.S.; visualization, C.K.; supervision, G.S. All authors have read and agreed to the published version of the manuscript.

Funding: This research received no external funding.

Acknowledgments: The present review was supported by the Hellenic Society of Medical Mycology.

Conflicts of Interest: The authors declare no conflict of interest.

\section{References}

1. Hébert-Seropian, S.; Pelet, S. Aspergillus Osteomyelitis of the Scapula: A Case Report. JBJS Case Connect. 2020, 10, e0343. [CrossRef]

2. Koutserimpas, C.; Chamakioti, I.; Naoum, S.; Raptis, K.; Alpantaki, K.; Kofteridis, D.P.; Samonis, G. Spondylodiscitis Caused by Aspergillus Species. Diagnostics 2021, 11, 1899. [CrossRef] [PubMed]

3. Latgé, J.P.; Chamilos, G. Aspergillus fumigatus and Aspergillosis in 2019. Clin. Microbiol. Rev. 2019, 33, e00140-18. [CrossRef]

4. Gautier, M.; Normand, A.C.; Ranque, S. Previously unknown species of Aspergillus. Clin. Microbiol. Infect. 2016, 22, 662-669. [CrossRef]

5. Samonis, G.; Koutserimpas, C.; Vrioni, G.; Kampos Martinez, E.; Kouloumentas, P.; Alpantaki, K.; Saroglou, G. Fungal Septic Knee Arthritis Caused by Aspergillus fumigatus following Anterior Cruciate Ligament Reconstruction. Diagnostics 2021, $11,1975$. [CrossRef] [PubMed]

6. Koutserimpas, C.; Chamakioti, I.; Zervakis, S.; Raptis, K.; Alpantaki, K.; Kofteridis, D.P.; Vrioni, G.; Samonis, G. Non-Candida Fungal Prosthetic Joint Infections. Diagnostics 2021, 11, 1410. [CrossRef] [PubMed]

7. Khalid, M.; Ali, S.A. Fungal osteomyelitis in a patient with chronic granulomatous disease: Case report and review of the literature. J. Pak. Med. Assoc. 2018, 68, 1387-1390.

8. Dotis, J.; Panagopoulou, P.; Filioti, J.; Winn, R.; Toptsis, C.; Panteliadis, C.; Roilides, E. Femoral osteomyelitis due to Aspergillus nidulans in a patient with chronic granulomatous disease. Infection 2003, 31, 121-124. [CrossRef] 
9. Hosalkar, H.S.; Gill, I.P.; Monsell, F.; Sau, I.; Ramsay, A. Hip pain in a 12-year-old boy. Clin. Orthop. Relat. Res. 2003, 416, 325-332. [CrossRef]

10. Bodur, H.; Ozoran, K.; Colpan, A.; Balaban, N.; Tabak, Y.; Kulacoglu, S. Arthritis and osteomyelitis due to Aspergillus fumigatus: A 17 years old boy with chronic granulomatous disease. Ann. Clin. Microbiol. Antimicrob. 2003, 2, 2. [CrossRef]

11. Shouldice, E.; Fernandez, C.; McCully, B.; Schmidt, M.; Fraser, R.; Cook, C. Voriconazole treatment of presumptive disseminated Aspergillus infection in a child with acute leukemia. J. Pediatr. Hematol. Oncol. 2003, 25, 732-734. [CrossRef]

12. Farhoudi, A.; Siadati, A.; Atarod, L.; Tabatabaei, P.; Mamishi, S.; Khotaii, G.; Armin, S.; Shirvani, F. Para Vertebral Abscess and Rib Osteomyelitis due to Aspergillous Fumigatus in a Patient with Chronic Granulomatous Disease. Iran. J. Allergy Asthma Immunol. 2003, 2, 13-15. [PubMed]

13. Salloum, A.; Rao, S.; Havasi, A.; Miljkovic, G.; Amoateng-Adjepong, Y. Aspergillus rib and vertebral osteomyelitis in a former intravenous drug user. Am. J. Med. 2004, 116, 208-209. [CrossRef]

14. Lodge, B.A.; Ashley, E.D.; Steele, M.P.; Perfect, J.R. Aspergillus fumigatus empyema, arthritis, and calcaneal osteomyelitis in a lung transplant patient successfully treated with posaconazole. J. Clin. Microbiol. 2004, 42, 1376-1378. [CrossRef] [PubMed]

15. Florio, M.; Marroni, M.; Morosi, S.; Stagni, G. Nosocomial Aspergillus flavus wound infections following cardiac surgery. Infez. Med. 2004, 12, 270-273. [PubMed]

16. Soto-Hurtado, E.J.; Marín-Gámez, E.; Segura-Domínguez, N.; Jiménez-Oñate, F. Pleural aspergillosis with bronchopleurocutaneous fistula and costal bone destruction: A case report. Lung 2005, 183, 417-423. [CrossRef] [PubMed]

17. Brodsky, J.W.; Seidenfeld, S.M.; Brooks, B.; Shabat, S. Aspergillus osteomyelitis and lymphangitis in immunocompromised patient after toenail clipping. Foot Ankle Int. 2005, 26, 576-578. [CrossRef]

18. Elahi, M.M.; Mitra, A.; Spears, J.; McClurken, J.B. Recalcitrant chest wall Aspergillus fumigatus osteomyelitis after coronary artery bypass grafting: Successful radical surgical and medical management. Ann. Thorac. Surg. 2005, 79, 1057-1059. [CrossRef] [PubMed]

19. Mamishi, S.; Zomorodian, K.; Saadat, F.; Gerami-Shoar, M.; Tarazooie, B.; Siadati, S.A. A case of invasive aspergillosis in CGD patient successfully treated with Amphotericin B and INF-gamma. Ann. Clin. Microbiol. Antimicrob. 2005, 4, 4. [CrossRef]

20. Mouas, H.; Lutsar, I.; Dupont, B.; Fain, O.; Herbrecht, R.; Lescure, F.X.; Lortholary, O.; Voriconazole/Bone Invasive Aspergillosis Study Group. Voriconazole for invasive bone aspergillosis: A worldwide experience of 20 cases. Clin. Infect. Dis. 2005, 40, 1141-1147. [CrossRef] [PubMed]

21. Ahmed, R. Disseminated aspergillosis causing spinal cord compression in a child. Neurol. India 2007, 55, 308-309. [CrossRef] [PubMed]

22. Scott, C.J.; Lambert, J.S.; Taylor, C.B.; Poulton, M.B. Invasive Aspergillus fumigatus associated with liver and bone involvement in a patient with AIDS. Int. J. Infect. Dis. 2007, 11, 550-553. [CrossRef] [PubMed]

23. Natesan, S.; Abraham, G.; Mathew, M.; Lalitha, M.K.; Srinivasan, C.N. Secondary sternal Aspergillus osteomyelitis in a diabetic hemodialysis patient with previous allograft rejection. Hemodial. Int. 2007, 11, 403-405. [CrossRef]

24. Ersoy, A.; Akdag, I.; Akalin, H.; Sarisozen, B.; Ener, B. Aspergillosis osteomyelitis and joint infection in a renal transplant recipient. Transplant. Proc. 2007, 39, 1662-1663. [CrossRef]

25. Dellepiane, R.M.; Tortorano, A.M.; Liotto, N.; Laicini, E.; Di Landro, G.; Carnelli, V.; Pietrogrande, M.C. Invasive Aspergillus nidulans infection in a patient with chronic granulomatous disease. Mycoses 2008, 51, 458-460. [CrossRef]

26. Ghotaslou, R.; Parvizi, R.; Safaei, N.; Yousefi, S. A case of Aspergillus fumigatus mediastinitis after heart surgery in Madani Heart Center, Tabriz, Iran. Prog. Cardiovasc. Nurs. 2008, 23, 133-135. [CrossRef]

27. Crassard, N.; Hadden, H.; Piens, M.A.; Pondarré, C.; Hadden, R.; Galambrun, C.; Pracros, J.P.; Souillet, G.; Basset, T.; Berthier, J.C.; et al. Invasive aspergillosis in a paediatric haematology department: A 15-year review. Mycoses 2008, 51, 109-116. [CrossRef]

28. Garazzino, S.; Maiello, A.; De Rosa, F.G.; Aprato, A.; Di Perri, G. Post-traumatic osteomyelitis due to Aspergillus flavus successfully treated with voriconazole: A case report. J. Chemother. 2008, 20, 524-526. [CrossRef]

29. Verghese, S.; Chellamma, T.; Cherian, K.M. Osteomyelitis of the rib caused by Aspergillus flavus following cardiac surgery. Mycoses 2009, 52, 91-93. [CrossRef]

30. Hodiamont, C.J.; Dolman, K.M.; Ten Berge, I.J.; Melchers, W.J.; Verweij, P.E.; Pajkrt, D. Multiple-azole-resistant Aspergillus fumigatus osteomyelitis in a patient with chronic granulomatous disease successfully treated with long-term oral posaconazole and surgery. Med. Mycol. 2009, 47, 217-220. [CrossRef]

31. Horn, D.; Sae-Tia, S.; Neofytos, D. Aspergillus osteomyelitis: Review of 12 cases identified by the Prospective Antifungal Therapy Alliance registry. Diagn. Microbiol. Infect. Dis. 2009, 63, 384-387. [CrossRef] [PubMed]

32. Winterstein, A.R.; Bohndorf, K.; Vollert, K.; Wagner, T.; Gnekow, A.; Roemer, F.W. Invasive aspergillosis osteomyelitis in children-a case report and review of the literature. Skeletal Radiol. 2010, 39, 827-831. [CrossRef]

33. Karia, S.; Jeyapalan, K.; Kennedy, B. Aspergillus fumigatus osteomyelitis in a patient receiving alemtuzumab for B-cell chronic lymphocytic leukaemia. Br. J. Haematol. 2011, 153, 147. [CrossRef] [PubMed]

34. Antkowiak, T.T.; Polage, C.R.; Wiedeman, J.A.; Meehan, J.P.; Jamali, A.A. Chondrolysis of the tibial plateau caused by articular aspergillosis after ACL autograft reconstruction: Management with a fresh osteochondral allograft: A case report. J. Bone Joint Surg. Am. 2011, 93, e124. [CrossRef] [PubMed]

35. Neild, G.H.; Jamale, T.E.; Hase, N.K.; Fernandez, G.C. A case of shoulder pain post-renal transplant: An unusual etiology. NDT Plus. 2011, 4, 136-137. 
36. Khemiri, M.; El Fekih, N.; Borgi, A.; Kharfi, M.; Boubaker, S.; Barsaoui, S. Pseudotumoral cutaneous aspergillosis in chronic granulomatous disease, report of a pediatric case. Am. J. Dermatopathol. 2012, 34, 749-752. [CrossRef] [PubMed]

37. Bassiri-Jahromi, S.; Doostkam, A. Fungal infection and increased mortality in patients with chronic granulomatous disease. J. Mycol. Med. 2012, 22, 52-57. [CrossRef] [PubMed]

38. Hall, G.L.; Villanueva-Siles, E.; Borzykowski, R.M.; Gruson, K.I.; Dorfman, H.D.; Geller, D.S. Aspergillus osteomyelitis of the proximal humerus: A case report. Skeletal Radiol. 2012, 41, 1021-1025. [CrossRef]

39. Asare, K.A.; Jahng, M.; Pincus, J.L.; Massie, L.; Lee, S.A. Sternal osteomyelitis caused by Aspergillus fumigatus following cardiac surgery: Case and review. Med. Mycol. Case Rep. 2012, 2, 4-6. [PubMed]

40. Mirhosseini, S.J.; Salehi, M.; Ali-Hassan-Sayegh, S.; Forouzannia, K.; Karimi-Bondarabadi, A.A. Costochondritis caused by Aspergillus flavus following cardiac surgery. Acta Med. Iran. 2013, 51, 733-735.

41. D'sa, S.R.; Singh, S.; Satyendra, S.; Mathews, P. Case report of Aspergillus osteomyelitis of the ribs in an immunocompetent patient J. Glob. Infect. Dis. 2013, 5, 118-120. [CrossRef]

42. Pattanashetty, O.B.; Bb, D.; Bhavi, S.B.; Bami, M. Rare case of Isolated Aspergillus Osteomyelitis of Toe: Presentation and Management. J. Orthop. Case Rep. 2013, 3, 29-31. [CrossRef] [PubMed]

43. Tiwari, V.; Khatri, K.; Khan, S.A.; Nath, D. Disseminated Aspergillus flavus following septic arthritis in an immunocompetent patient: A case report. BMC Res. Notes 2014, 7, 709. [CrossRef]

44. Dabkana, T.M.; Pindiga, U.H.; Mayun, A.A.; Nggada, H.A. Aspergillosis of the bone. Ann. Afr. Med. 2015, 14, 62-64. [CrossRef] [PubMed]

45. Domenech, C.; Leick-Courtois, C.; Bienvenu, A.L.; Pracros, J.P.; Picot, S.; Bleyzac, N.; Bertrand, Y. Improvement in the Outcome of Invasive Aspergillosis in a Pediatric Hematology Department: A 10-Year Review. J. Pediatr. Hematol. Oncol. 2015, 37, $560-565$. [CrossRef]

46. Babamahmoodi, F.; Shokohi, T.; Ahangarkani, F.; Nabili, M.; Afzalian Ashkezari, E.; Alinezhad, S. Rare Case of Aspergillus ochraceus Osteomyelitis of Calcaneus Bone in a Patient with Diabetic Foot Ulcers. Case Rep. Med. 2015, 2015, 509827. [CrossRef] [PubMed]

47. Uzodi, A.S.; Tebben, P.J.; Boyce, T.G. Bone Pain in a 4-year-old Boy with Chronic Granulomatous Disease and History of Aspergillus pneumonia. Pediatr. Infect. Dis. J. 2016, 35, 464-465, 469. [CrossRef]

48. Mamishi, S.; Zomorodian, K.; Saadat, F.; Jalali, S.Z.; Geramishoar, M. Osteomyelitis and lung abscess due to Aspergillus fumigatus in a chronic granulomatous disease patient. Curr. Med. Mycol. 2016, 2, 37-41. [CrossRef]

49. Menon, A.; Rodrigues, C.; Soman, R.; Sunavala, A.; Agashe, V.M. Aspergillus Osteomyelitis of the Ribs in Immunocompetent Hosts: Report of Two Rare Cases. J. Orthop. Case Rep. 2017, 7, 61-64.

50. Kutluğ, Ş.; Şensoy, G.; Birinci, A.; Saraymen, B.; Yavuz Köker, M.; Yıldıran, A. Seven chronic granulomatous disease cases in a single-center experience and a review of the literature. Asian Pac. J. Allergy Immunol. 2018, 36, 35-41.

51. Uehara, Y.; Kasai, H.; Nakajima, T.; Tanabe, N.; Tatsumi, K.; Yoshino, I. Aspergillus Sternomyelitis Developed from Chronic Pulmonary Aspergillosis as a Late Complication to Lobectomy for Lung Cancer. Intern. Med. 2018, 57, 2991-2994. [CrossRef]

52. Landaburu, M.F.; López Daneri, G.; PLoszaj, F.; Kruss, M.; Vinante, A.; Veciño, C.H.; Mujica, M.T. Osteomyelitis of the rib cage by Aspergillus flavus. Rev. Iberoam. Micol. 2019, 36, 86-89. [CrossRef] [PubMed]

53. Assaf, A.; Faure, E.; Sermet, K.; Loridant, S.; Leroy, J.; Goeminne, C.; Dozier, A.; Chopin, M.C.; Panaget, S.; Faure, K.; et al. Successful treatment of Aspergillus fumigatus sternal osteomyelitis with isavuconazole in a heart transplant recipient. Transpl. Infect. Dis. 2020, 22, e13313. [CrossRef]

54. Doub, J.B.; Mathur, P. Duration of posaconazole therapy for Aspergillus fumigatus osteomyelitis dictated by serial monitoring of 1,3-beta-D glucan. Infection 2020, 48, 959-963. [CrossRef] [PubMed]

55. Tavakoli, M.; Hedayati, M.T.; Mirhendi, H.; Nouripour-Sisakht, S.; Hedayati, N.; Saghafi, F.; Mamishi, S. The first rare and fatal case of invasive aspergillosis of spinal cord due to Aspergillus nidulans in an Iranian child with chronic granulomatosis disease: Review of literature. Curr. Med. Mycol. 2020, 6, 55-60. [CrossRef]

56. Routray, C.; Nwaigwe, C. Sternal osteomyelitis secondary to Aspergillus fumigatus after cardiothoracic surgery. Med. Mycol. Case Rep. 2020, 28, 16-19. [CrossRef]

57. Ordaya, E.E.; Johnson, J.R.; Drekonja, D.M.; Niehans, G.E.; Kaka, A.S. Aspergillus Osteomyelitis Secondary to Chronic Necrotizing Pulmonary Aspergillosis in a Patient With Rheumatoid Arthritis. Cureus 2021, 13, e17774. [CrossRef]

58. Pérez-Cantero, A.; López-Fernández, L.; Guarro, J.; Capilla, J. Azole resistance mechanisms in Aspergillus: Update and recent advances. Int. J. Antimicrob. Agents 2020, 55, 105807. [CrossRef] [PubMed]

59. Gamaletsou, M.N.; Rammaert, B.; Bueno, M.A.; Sipsas, N.V.; Moriyama, B.; Kontoyiannis, D.P.; Roilides, E.; Zeller, V.; Taj-Aldeen, S.J.; Henry, M.; et al. Aspergillus arthritis: Analysis of clinical manifestations, diagnosis, and treatment of 31 reported cases. Med. Mycol. 2017, 55, 246-254. [PubMed]

60. Herbrecht, R.; Denning, D.W.; Patterson, T.F.; Bennett, J.E.; Greene, R.E.; Oestmann, J.W.; Kern, W.V.; Marr, K.A.; Ribaud, P.; Lortholary, O.; et al. Voriconazole versus amphotericin B for primary therapy of invasive aspergillosis. N. Engl. J. Med. 2002, 347, 408-415. [CrossRef] [PubMed]

61. Cadena, J.; Thompson, G.R., 3rd; Patterson, T.F. Aspergillosis: Epidemiology, Diagnosis, and Treatment. Infect. Dis. Clin. N. Am. 2021, 35, 415-434. [CrossRef] 
62. Nett, J.E.; Andes, D.R. Antifungal Agents: Spectrum of Activity, Pharmacology, and Clinical Indications. Infect. Dis. Clin. N. Am. 2016, 30, 51-83. [CrossRef] [PubMed]

63. Koutserimpas, C.; Zervakis, S.G.; Maraki, S.; Alpantaki, K.; Ioannidis, A.; Kofteridis, D.P.; Samonis, G. Non-albicans Candida prosthetic joint infections: A systematic review of treatment. World J. Clin. Cases 2019, 7, 1430-1443. [CrossRef] [PubMed]

64. Koutserimpas, C.; Samonis, G.; Velivassakis, E.; Iliopoulou-Kosmadaki, S.; Kontakis, G.; Kofteridis, D.P. Candida glabrata prosthetic joint infection, successfully treated with anidulafungin: A case report and review of the literature. Mycoses 2018, 61, 266-269. [CrossRef] [PubMed]

65. Anwer, U.; Yablon, C.M. Imaging of Osteomyelitis of the Extremities. Semin. Roentgenol. 2017, 52, 49-54. [CrossRef]

66. Tashiro, M.; Izumikawa, K.; Minematsu, A.; Hirano, K.; Iwanaga, N.; Ide, S.; Mihara, T.; Hosogaya, N.; Takazono, T.; Morinaga, Y.; et al. Antifungal susceptibilities of Aspergillus fumigatus clinical isolates obtained in Nagasaki, Japan. Antimicrob. Agents Chemother. 2012, 56, 584-587. [CrossRef]

67. Zhang, J.; Debets, A.J.M.; Verweij, P.E.; Snelders, E. Azole-Resistance Development; How the Aspergillus fumigatus Lifecycle Defines the Potential for Adaptation. J. Fungi 2021, 7, 599. [CrossRef]

68. Lamoth, F.; Lewis, R.E.; Kontoyiannis, D.P. Role and Interpretation of Antifungal Susceptibility Testing for the Management of Invasive Fungal Infections. J. Fungi 2020, 7, 17. [CrossRef]

69. Arendrup, M.C.; Friberg, N.; Mares, M.; Kahlmeter, G.; Meletiadis, J.; Guinea, J.; Subcommittee on Antifungal Susceptibility Testing (AFST) of the ESCMID European Committee for Antimicrobial Susceptibility Testing (EUCAST). How to interpret MICs of antifungal compounds according to the revised clinical breakpoints v. 10.0 European committee on antimicrobial susceptibility testing (EUCAST). Clin. Microbiol. Infect. 2020, 26, 1464-1472. [CrossRef]

70. Hamill, R.J. Amphotericin B formulations: A comparative review of efficacy and toxicity. Drugs 2013, 73, 919-934. [CrossRef] 